\title{
Repetition of chemistry from a recently retracted paper. A cautionary note.
}

Wilson Ryan, ${ }^{\text {a }}$ Korey Bedard, ${ }^{a}$ Daler Baidilov, ${ }^{\text {a }}$ Marcus Tius ${ }^{\mathrm{b}}$ and Tomas Hudlicky ${ }^{\mathrm{a} *}$

a. Department of Chemistry and Centre for Biotechnology, Brock University, 1812 Sir Isaac Brock Way, St. Catharines, ON L2S 3A1, Canada thudlicky@brocku.ca

b. Department of Chemistry, University of Hawaii at Manoa, 2545 McCarthy Mall Honolulu, HI 96822-2275

\begin{abstract}
The base-catalyzed condensation reaction between $(E)$-4-phenylbut-2-enal and phenylpropargyl aldehyde recently reported in the literature to provide formylcyclobutadiene was repeated under the published conditions. The product obtained was identified as $(E)$-5-phenyl-2-((E)-styryl)pent-2-en-4-ynal rather than the reported 2phenyl-3-styrylcyclobutadiene-1-carboxaldehyde. The structure assignment is supported by NMR and IR data and a x-ray structure of the crystalline alcohol obtained by Luche reduction.
\end{abstract}

The strict requirements for physical reproduction of research results ensure reproducibility and provide lasting experimental value to the community. In today's world of rapid and exponentially increasing rates of publication both the reproducibility of results and the overall value to the permanent record are jeopardized. In addition, characterization of compounds, often incomplete, at small scales can (and does) lead to problems in structure assignments.

In August 2017 a paper published in Org. Lett. ${ }^{1}$ reported the synthesis of a series of cyclobutadienes, as shown in Figure 1. One of us (MT) was struck by the implausibility of the cyclobutadiene enal structures. Even if the anti-aromatic compounds had been prepared, the highly reactive formylcyclobutadiene would surely not have survived for long. Also, the ${ }^{13} \mathrm{C}$ NMR spectra reported for the cyclobutadiene products contained signals a 109.62 and 86.91 ppm (compound 3aa) that would be more appropriately assigned to disubstituted alkynes rather than to cyclobutadienes.

In addition, the anti-aromatic cyclobutadienes are not stable unless coordinated to metals (this fact was also mentioned and referenced by the authors) ${ }^{2}$ and these observations led 
to our questioning of the reported results. We decided to repeat the chemistry and provide experimental validation of the products.

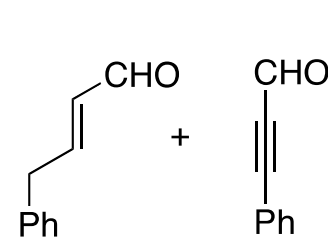

1

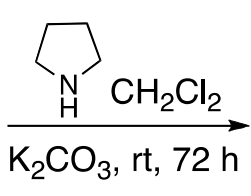

$(58 \%)$

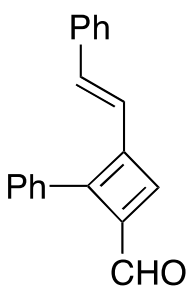

3

[3aa in reference 1,17 other examples]

Figure 1. Reported synthesis of cyclobutadienes. ${ }^{1}$

While we were repeating the experimental protocol the above paper was retracted ${ }^{3}$ in December 2017, citing as the reason inadequate characterization of the products by ${ }^{13} \mathrm{C}$ NMR. We continued our work and have completed the project for reasons of academic interest and also to provide the results in this short note that should serve as a cautionary reminder to authors and referees of the importance of validating chemical structures with complete experimental and spectral data sets.

In our repetition we chose the substrates shown in Figure 1 and obtained aldehyde $\mathbf{4}$ in $58 \%$ yield [the reported yield in the literature for compound 3aa was 58\% under the conditions shown in Figure 1]. Under different conditions and with a different organocatalyst (one derived from proline) the authors reported a yield of $87 \%$ for 3aa.

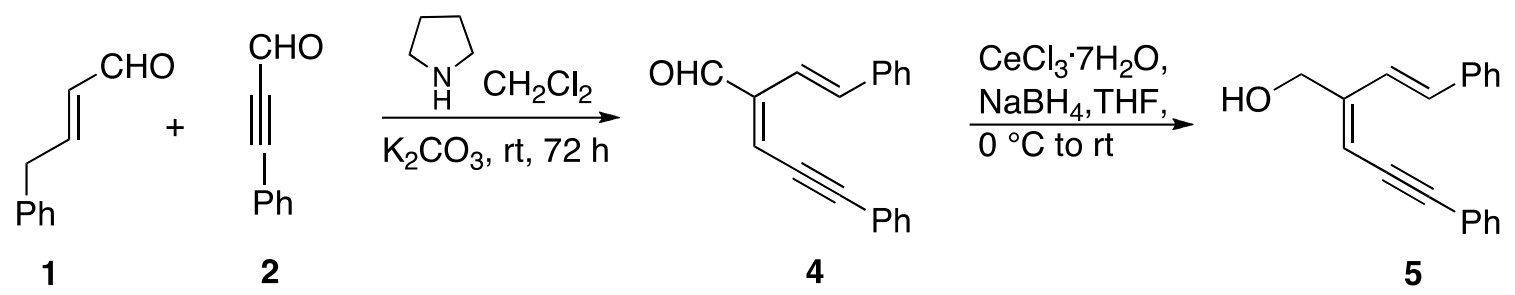

Figure 2. Repetition of the condensation reaction of $\mathbf{1}$ and $\mathbf{2}$.

Reduction of this somewhat unstable aldehyde provided alcohol 5 [white needles, mp 119-120 ${ }^{\circ} \mathrm{C}$, EtOH: $\left.\mathrm{H}_{2} \mathrm{O}, 75: 25\right]$ whose structure, along with that of aldehyde 4, was validated by detailed NMR analysis (See the Supplemental Information Section for data). Both compounds showed acetylene absorptions in the IR spectrum (4 at $2183 \mathrm{~cm}^{-1}$ and 5 at $2030 \mathrm{~cm}^{-1}$ ). IR analysis was not reported in the Experimental Section of the original paper and this fact alone would have cast doubt on the assignment of products as cyclobutadienes as the IR absorptions clearly indicate the presence of an acetylene. 
Figure 3 shows the ${ }^{13} \mathrm{C}$ NMR data for compound $\mathbf{4}$ as obtained by us compared with the ${ }^{13} \mathrm{C}$ NMR published data of 3aa. Although the signal for one carbon atom is missing in the published spectrum it is clear that the two compounds are identical.

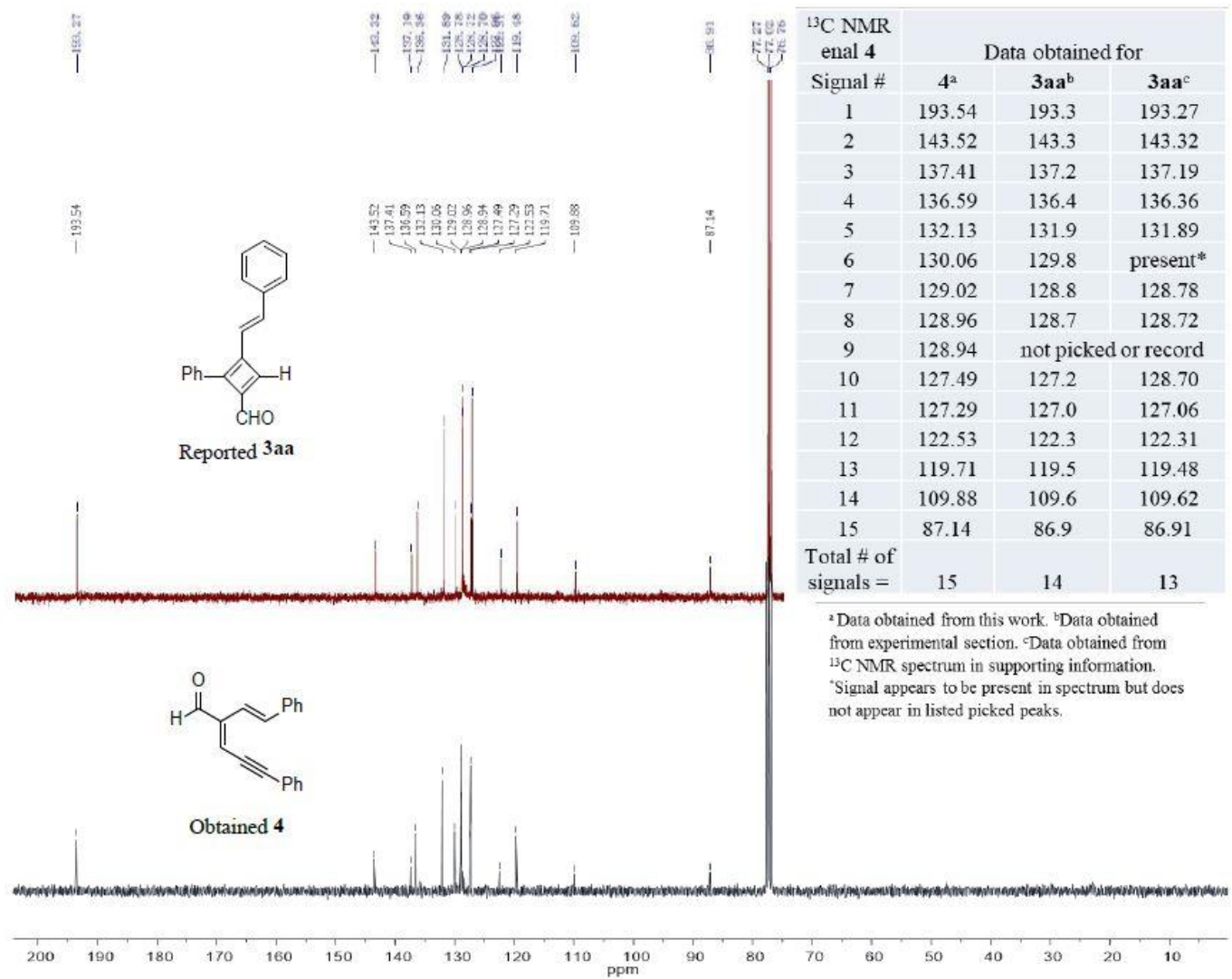

Figure 3. Comparison of ${ }^{13} \mathrm{C}$ NMR spectrum of $\mathbf{4}$ with that of compound 3aa as reported in the literature. ${ }^{1}$ (Scale of our spectrum (see SI section) was adjusted for comparison.) Alcohol 5 was converted to its $p$-bromobenzoate in an effort to produce crystalline material for x-ray analysis, however, the ester was a low melting solid. Finally, suitable crystals for x-ray analysis were obtained from alcohol 5, as shown in Figure $4 .^{4}$

The take home lesson form this exercise is clear: more attention should be paid to the reported experimental data and their integrity. It should have been noticed by the referees that the authors did not obtain/report IR spectra for any of the compounds. Relying solely on NMR and MS data is obviously insufficient. While we repeated only the synthesis of the presumed 3aa it is likely that none of the other compounds reported are cyclobutadienes, as evidenced by the signals (corresponding to acetylenes) in their ${ }^{13} \mathrm{C}$ NMR spectra. Perhaps a more rigorous attention to review may eliminate similar 
occurrences in the future. We enjoyed this particular exercise but one has to wonder how many other erroneous results are contained in today's literature.

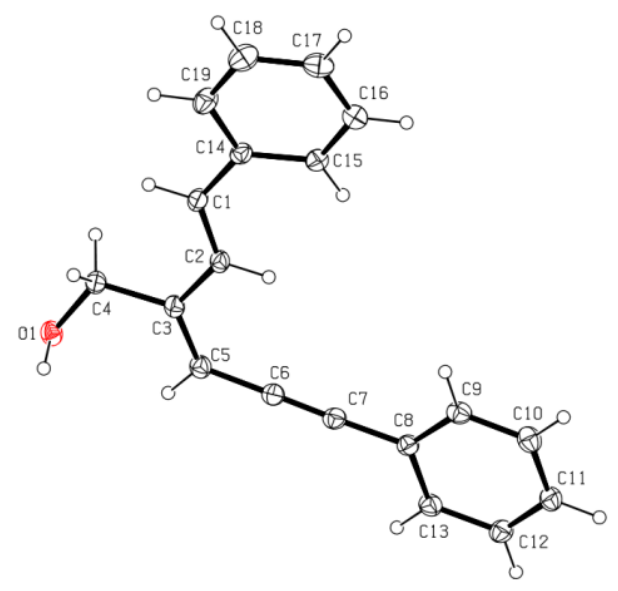

Figure 4. X-ray structure of 5.

Acknowledgements. We are grateful to the following agencies for financial support of this work: Natural Sciences and Engineering Research Council of Canada (NSERC) (Idea to Innovation and Discovery Grants), Canada Research Chair Program, Canada Foundation for Innovation (CFI), TDC Research, Inc., TDC Research Foundation.

Supplemental Information Section: Experimental and spectral data for the synthesis and characterization of compounds $\mathbf{4}$, and $\mathbf{5}$.

\section{References}

1.Li, W.; Lang, M.; Wang, J. Org. Lett. 2017, 19, 4564.

2.(a) Emerson, G. F.; Watts, L.; Pettit, R. J. Am. Chem. Soc. 1965, 87, 131; (b) Watts, L.; Fitzpatrick, J. D.; Pettit, R. J. Am. Chem. Soc. 1965, 87, 3253; (c) Rosenblum, M.; Gatsonis, C. J. Am. Chem. Soc. 1967, 89, 5074; (d) Sekiguchi, A.; Tanaka, M.; Matsuo, T.; Watanabe, H. Angew. Chem., Int. Ed. 2001, 40, 1675.

3. Li, W.; Lang, M.; Wang, J. Org. Lett. 2018, 20, 316.

4. While this work was being completed a recent publication also reported the synthesis of enal 4 obtained by repetition of the work in reference 1: Riveira, M. J.; Sarotti, A.M. Org. Biomol. Chem. 2018, 16, 1442. The structure was elucidated by NMR methods only. The unambiguous assignment of the alcohol 5 by x-ray is provided in this work, which was submitted originally to Org. Lett. but was rejected by the Editor without external review. 